\title{
HOMOLOGICAL DIMENSION IN NOETHERIAN RINGS. II ${ }^{(1)}$
}

\author{
BY \\ MAURICE AUSLANDER AND DAVID A. BUCHSBAUM
}

Introduction. Throughout this paper it is assumed that all rings are commutative, noetherian rings with unit element and all modules are unitary. The major purpose of this paper is to extend to arbitrary noetherian rings the homological invariants which were introduced in [2] for local rings.

In $\S 1$ we study the codimension of modules, and prove, in particular, that if $E$ is any finitely generated $R$-module, then

$$
\operatorname{codim}_{R} E=\sup _{\mathfrak{m}} \operatorname{codim}_{R_{\mathfrak{m}}} E_{\mathfrak{m}}
$$

where $\mathrm{m}$ runs through all maximal ideals of $R, R_{\mathrm{m}}$ is the local ring of quotients of $R$ with respect to $\mathfrak{m}$, and $E_{\mathfrak{m}}=R_{\mathfrak{m}} \otimes_{R} E$. We also show that the result which we obtained in [2],

$$
\operatorname{codim} R=\sup _{E} \operatorname{hd}_{R} E
$$

where $E$ runs through all finitely generated $R$-modules of finite homological dimension, holds when $R$ is any noetherian ring.

Another result obtained in this section is that if $R\left[\left[X_{1}, \cdots, X_{n}\right]\right]=S$ is the ring of formal power series over $R$, then

$$
\begin{aligned}
& \operatorname{codim}_{S} S=n+\operatorname{codim}_{R} R, \\
& \text { gl. } \operatorname{dim} S=n+\text { gl. } \operatorname{dim} R .
\end{aligned}
$$

In $\$ 2$ we introduce the weak homological dimension of an $R$-module $E$ (w. $\operatorname{hd}_{R} E$ ). It is defined as the smallest integer $n$ (or $+\infty$ ) such that $\operatorname{Tor}_{p}^{R}(E, C)=0$ for all $p>n$, and all $R$-modules $C$. The finitistic weak global dimension of $R$ is defined by

$$
\text { f.w. gl. } \operatorname{dim} R=\sup _{E} \text { w. } \operatorname{hd}_{R} E
$$

where $E$ runs over all modules of finite weak homological dimension. We then show that

$$
\text { f.w. gl. } \operatorname{dim} R=\sup _{\mathfrak{p}} \operatorname{codim}_{R_{\mathfrak{p}}} R_{\mathfrak{p}} \leqq \operatorname{dim} R
$$

Received by the editors October 31, 1956.

(1) This work was done while one of the authors was on a National Science Foundation Fellowship. 
where $\mathfrak{p}$ runs through all prime ideals in $R$. For polynomial rings $R[x]$ over $R$, we obtain the equalities

$$
\text { f.w. gl. } \operatorname{dim} R[x]=\operatorname{codim}_{R[x]} R[x]=1+\text { f.w. gl. } \operatorname{dim} R .
$$

An example is given to show that $\operatorname{codim}_{R[x]} R[x]$ can be arbitrarily larger than $\operatorname{codim}_{R} R$.

$\$ 3$ deals with the question of unique factorization in local rings. It has been communicated to the authors that some of the results we establish in this section have been proven (but not yet published) by Mori. However, the methods used in our proofs are homological and, we feel, are of sufficient interest to be included in this paper.

The main result of the section is that a local integral domain $R$ is a unique factorization domain if and only if $h_{R} R /(x, y) \leqq 2$ for every pair of elements $x, y \in R$. From this it follows that $R$ is a unique factorization domain if its completion, $\hat{R}$, is. We thereby conclude that every nonramified regular local ring is a unique factorization domain.

The terminology used throughout is the same as that used in [2]. The notation used is standard except for the following situation:

If $S$ is a multiplicatively closed subset of the ring $R$, not containing zero, and $E$ is an $R$-module, we denote by $E_{S}$ the $R_{S}$-module $R_{S} \otimes_{R} E$. However, if $S=R-\mathfrak{p}$, where $\mathfrak{p}$ is a prime, we write $E_{\mathfrak{p}}$ instead of $E_{S}$.

1. Codimension. Throughout this section we assume that all modules are finitely generated.

An element $x$ in the ring $R$ is said to be a zero divisor for the $R$-module $E$ if there is a nonzero element $e$ in $E$ such that $x e=0$. A finite sequence $x_{1}, \cdots, x_{s}$ of elements in $R$ is called an $E$-sequence if $x_{i}$ is not a zero divisor for the module $E /\left(x_{1}, \cdots, x_{i-1}\right) E$ and $E /\left(x_{1}, \cdots, x_{s}\right) E \neq 0$. Given an ideal $\mathfrak{f}$ in $R$, an $E$-sequence is said to be a maximal E-sequence in $\mathfrak{f}$ if $\mathfrak{t}$ contains $\left(x_{1}, \cdots, x_{s}\right)$ and given any $y$ in $f$, the sequence $x_{1}, \cdots, x_{8}, y$ is not an $E$ sequence. It is clear that if $x_{1}, \cdots, x_{s}$ is an $E$-sequence then $\left(x_{1}, \cdots, x_{i}\right)$ $\neq\left(x_{1}, \cdots, x_{i+1}\right)$ for any $i$. Since $R$ is a noetherian ring, it follows that each $E$-sequence in $\mathfrak{f}$ can be imbedded in a maximal $E$-sequence in $\mathfrak{f}$ of finite length. If $x_{1}, \cdots, x_{s}$ is a maximal $E$-sequence in $\mathfrak{l}=R$, we shall simply say that $x_{1}, \cdots, x_{8}$ is a maximal $E$-sequence. The least upper bound of lengths of $E$-sequences (finite or $+\infty$ ) is called the codimension of $E$ (notation: $\left.\operatorname{codim}_{R} E\right)$.

Lemma 1.1. Let $E$ be an $R$-module and $\mathfrak{p}$ a prime ideal containing the annihilator of $E$. If $x_{1}, \cdots, x_{8}$ is an E-sequence contained in $\mathfrak{p}$, then $\mathfrak{p}$ contains the annihilator of $E /\left(x_{1}, \cdots, x_{s}\right) E$.

Proof. The proof proceeds by induction on $s$. If $s=0$, there is nothing to prove. Suppose lemma true for $s=n \geqq 0$ and let $s=n+1$. By the induction hypothesis $\mathfrak{p}$ contains $\mathfrak{f}$, the annihilator of $E /\left(x_{1}, \cdots, x_{n}\right) E$. Thus $\mathfrak{p}$ contains 
some prime deal $\mathfrak{p}^{\prime}$ belonging to $\mathfrak{l}$. Since $x_{n+1}$ in $\mathfrak{p}$ is not a zero-divisor for $E /\left(x_{1}, \cdots, x_{n}\right) E$, it follows from $[2,1.2]$ that $\mathfrak{p}$ contains a prime ideal belonging to the annihilator of $E /\left(x_{1}, \cdots, x_{n+1}\right) E$. Therefore $p$ contains the annihilator of $E /\left(x_{1}, \cdots, x_{n+1}\right) E$.

Proposition 1.2. Let $E$ be an $R$-module, $m$ a maximal ideal in $R$ containing the annihilator of $E$, and $x_{1}, \cdots, x_{s}$ an E-sequence contained in $\mathrm{m}$. Then the following statements are equivalent:

(a) $x_{1}, \cdots, x_{s}$ is a maximal E-sequence in $\mathrm{m}$.

(b) $m$ belongs to (0) in $E /\left(x_{1}, \cdots, x_{s}\right) E$.

(c) The sequence $x_{1}, \cdots, x_{s}$, considered as elements in $R_{\mathfrak{m}}$, is a maximal $E_{\mathrm{m}}$-sequence.

(d) $\operatorname{codim}_{R_{\mathrm{m}}} E_{\mathfrak{l l}}=s$.

Proof. (a) $\Rightarrow(\mathrm{b})$. Suppose $\mathfrak{m}$ does not belong to $(0)$ in $E /\left(x_{1}, \cdots, x_{8}\right) E$. Since $\mathfrak{m}$ is a maximal ideal in $R$, we have that $\mathfrak{m}$ is not contained in any prime ideal belonging to $(0)$ in $E /\left(x_{1}, \cdots, x_{s}\right) E$. Thus there is a $y$ in $m$ which is not a zero-divisor for $E /\left(x_{1}, \cdots, x_{s}\right) E$. Since $m$ contains $x_{1}, \cdots, x_{s}$ and the annihilator of $E$, it follows from 1.1 that $m$ contains the annihilator of $E /\left(x_{1}, \cdots, x_{s}\right) E$. Consequently, $\mathfrak{m}$ contains a prime ideal $\mathfrak{p}$ belonging to $(0)$ in $E /\left(x_{1}, \cdots, x_{s}\right) E$. Therefore $\mathfrak{m}$ contains $(\mathfrak{p}, y)$ and thus by $[2,1.2]$ we have that $E /\left(x_{1}, \cdots, x_{s}, y\right) E \neq 0$. This contradicts the maximal nature of $x_{1}, \cdots, x_{s}$.

(b) $\Rightarrow$ (c). From the exact sequence of $R$-modules

$$
0 \rightarrow E /\left(x_{1}, \cdots, x_{i-1}\right) E \stackrel{f}{\rightarrow} E /\left(x_{1}, \cdots, x_{i-1}\right) E \rightarrow E /\left(x_{1}, \cdots, x_{i}\right) E \rightarrow 0
$$

we deduce the exact sequence of $R_{\mathfrak{m}}$-modules

$0 \rightarrow E_{\mathfrak{m}} /\left(x_{1}, \cdots, x_{i-1}\right) E_{\mathfrak{m}} \stackrel{g}{\rightarrow} E_{\mathfrak{m}} /\left(x_{1}, \cdots, x_{i-1}\right) E_{\mathfrak{m}} \rightarrow E_{\mathfrak{m}} /\left(x_{1}, \cdots, x_{i}\right) E_{\mathfrak{m}} \rightarrow 0$

where $f$ is multiplication by $x_{i}$ and $g$ is multiplication by the image of $x_{i}$ in $R_{\mathfrak{m}}$ which we shall denote also by $x_{i}$. Thus $x_{i}$ is not a zero-divisor for $E_{\mathfrak{m}} /\left(x_{1}, \cdots, x_{i-1}\right) E_{\mathfrak{m}}$. Since $\mathfrak{m}$ belongs to $(0)$ in $E /\left(x_{1}, \cdots, x_{8}\right) E$, we have by the general theory of noetherian modules [see 2,1.1] that $E_{\mathfrak{m}} /\left(x_{1}, \cdots, x_{s}\right) E_{\mathfrak{m}}$ $\neq 0$ and that the maximal ideal $\mathfrak{m} R_{\mathfrak{m}}$ of the local ring $R_{\mathfrak{m}}$ belongs to (0) in $E_{\mathfrak{m}} /\left(x_{1}, \cdots, x_{s}\right) E_{\mathfrak{m}}$. It follows therefore that $x_{1}, \cdots, x_{s}$ in $R_{\mathfrak{m}}$ is a maximal $E_{\mathfrak{m}}$-sequence.

(c) $\Rightarrow$ (d). This follows immediately from the fact that in a local ring all maximal sequences for a module have the same length [see 2, 3.4].

$(\mathrm{d}) \Rightarrow(\mathrm{a})$. Suppose $x_{1}, \cdots, x_{s}$ is not a maximal $E$-sequence in $\mathfrak{m}$. Then there is an $E$-sequence $x_{1}, \cdots, x_{s}, x_{s+1}, \cdots, x_{n}$ which is a maximal $E$ sequence in $\mathrm{m}$. Applying the implication (a) $\Rightarrow(\mathrm{d})$ we have $\operatorname{codim}_{R_{\mathfrak{m}}} E_{\mathfrak{m}}$ $=n>s$, which is a contradiction. 
Proposition 1.3. Let $E$ be an $R$-module and let the ideal $t$ in $R$ be the annihilator of $E$. If $x_{1}, \cdots, x_{s}$ in $R$ is an E-sequence, then the ideal $\left(\mathfrak{f}, x_{1}, \cdots, x_{s}\right) \neq R$.

Proof. Suppose $\left(\mathfrak{f}, x_{1}, \cdots, x_{s}\right)=R$. Then there exist $x$ in $\mathfrak{f}$ and $y$ in $\left(x_{1}, \cdots, x_{s}\right)$ such that $x+y=1$. Therefore $y e=e$ for all $e$ in $E$ which means that $E /\left(x_{1}, \cdots, x_{s}\right) E=0$. This contradicts the fact that $x_{1}, \cdots, x_{s}$ is an $E$-sequence.

Theorem 1.4. Let $E$ be an R-module. Then

$$
\operatorname{codim}_{R} E=\sup _{\mathfrak{m}} \operatorname{codim}_{R_{\mathfrak{m}}} E_{\mathfrak{m}}
$$

where $m$ runs through all maximal ideals in $R$.

Proof. Let $\mathfrak{m}$ be a maximal ideal in $R$. If $\mathfrak{m}$ does not contain the annihilator of $E$, then $E_{\mathfrak{m}}=0$ and thus $\operatorname{codim}_{R} E \geqq \operatorname{codim}_{R_{\mathfrak{m}}} E_{\mathfrak{m}}$. If $\mathfrak{m}$ does contain the annihilator of $E$, then by 1.2 we have that all maximal $E$-sequences in $\mathrm{m}$ have the same length, namely $\operatorname{codim}_{R_{\mathfrak{m}}} E_{\mathfrak{m}}$. Thus we have

$$
\operatorname{codim}_{R} E \geqq \sup _{\mathfrak{m}} \operatorname{codim}_{R_{\mathfrak{m}}} E_{\mathfrak{m}} \text {. }
$$

Suppose $x_{1}, \cdots, x_{s}$ in $R$ is a maximal $E$-sequence. Then by 1.3 we know there is a maximal ideal $\mathfrak{m}$ containing $x_{1}, \cdots, x_{s}$ and the annihilator of $E$. Since $x_{1}, \cdots, x_{s}$ is a maximal $E$-sequence it is maximal in $m$. We then have by 1.2 that $\operatorname{codim}_{R_{\mathfrak{m}}} E_{\mathfrak{m}}=s$. Therefore we have $\operatorname{codim}_{R} E \leqq \sup _{\mathfrak{m}} \operatorname{codim}_{R_{\mathfrak{m}}} E_{\mathfrak{m}}$.

Corollary 1.5. Let E be an R-module. Then

$$
\operatorname{codim}_{R} E \leqq \operatorname{dim} R \text {. }
$$

By $[2,1.3]$ we know that $\operatorname{codim}_{R_{\mathfrak{m}}} E_{\mathfrak{m}} \leqq \operatorname{dim} R_{\mathfrak{m}} \leqq \operatorname{dim} R$. Therefore it follows from 1.4 that $\operatorname{codim}_{R} E \leqq \operatorname{dim} R$.

TheOREM 1.6. For a ring $R$ we have

(a) $\operatorname{codim}_{R} R \leqq \operatorname{dim} R$,

(b) $\operatorname{codim}_{R} R=\operatorname{supm}_{\operatorname{m}} \operatorname{codim}_{R_{\mathfrak{m}}} R_{\mathfrak{m}}$,

(c) $\operatorname{codim}_{R} R=\sup _{E} h d_{R} E$,

where $m$ runs through all maximal ideals in $R$ and $E$ runs through all finitely generated $R$-modules such that $h d_{R} E<\infty$.

Proof. The first two relations are obtained by substituting $R$ for $E$ in 1.5 and 1.4 respectively.

Suppose $E$ is an $R$-module such that $\operatorname{hd}_{R} E<\infty$. Then we have by [3, VII, Exer. 11] that hd $R=\operatorname{supm}_{R} \mathrm{hd}_{R_{\mathfrak{m}}} E_{\mathfrak{m}}$, where $\mathrm{m}$ runs through all maximal ideals in $R$. Since for each $\mathrm{m}$ we have that $R_{\mathfrak{m}}$ is a local ring and $\mathrm{hd}_{R_{\mathfrak{m}}} E_{\mathfrak{m}}<\infty$, it follows from $[2,1.7]$ that $\mathrm{hd}_{R_{\mathfrak{m}}} E_{\mathfrak{m}} \leqq \operatorname{codim} R_{\mathfrak{m}}$ for all $\mathrm{m}$. From this we conclude that $\operatorname{hd}_{R} E \leqq \sup _{\mathfrak{m}} \operatorname{codim}_{R_{\mathfrak{m}}} R_{\mathfrak{m}}=\operatorname{codim} R$. On the other hand if 
$x_{1}, \cdots, x_{8}$ is an $R$-sequence, then $\operatorname{hd}_{R} R /\left(x_{1}, \cdots, x_{8}\right)=s$ (see [3, VIII, $4.2])$. Thus the last equality is established.

Corollary 1.7. If gl. $\operatorname{dim} R<\infty$, then

$$
\operatorname{codim}_{R} R=g l . \operatorname{dim} R=\operatorname{dim} R .
$$

By [1, Theorem 1] we know that gl. $\operatorname{dim} R=\sup _{E} \mathrm{hd}_{R} E$ where $E$ runs through all finitely generated $R$-modules. It follows therefore from 1.6 that $\operatorname{codim}_{R} R=$ gl. $\operatorname{dim} R$. The second equality was proven in $[2,4.7]$.

Proposition 1.8. Let $S$ be the factor ring $R / \mathfrak{f}$, where $\mathfrak{l}$ is an ideal in $R$. Suppose that $h d_{R} S<\infty$ and that $E$ is an $S$-module such that $h d_{S} E<\infty$. Then we have

$$
h d_{S} E \leqq h d_{R} E<\infty
$$

and thus

$$
\operatorname{codim}_{S} S \leqq \operatorname{codim}_{R} R .
$$

If we assume in addition that rank $\mathfrak{1}>0$, then we have

$$
h d_{S} E<h d_{R} E<\infty
$$

and thus ${ }^{(2)}$

$$
\operatorname{codim}_{S} S<\operatorname{codim}_{R} R \text {. }
$$

Proof. By [3, VII, Exercise 11] we know that there is a maximal prime ideal $\mathfrak{m}^{\prime}$ in $S$ such that $\mathrm{hd}_{S} E=\mathrm{hd}_{S_{\mathfrak{m}^{\prime}}} E_{\mathfrak{m}^{\prime}}$. Let $\mathfrak{m}$ be the preimage of $\mathfrak{m}^{\prime}$ in $R$ under the natural homomorphism $R \rightarrow S$. Clearly $S_{\mathfrak{m}^{\prime}}=S_{\mathfrak{m}}$ and $E_{\mathfrak{m}^{\prime}}=E_{\mathfrak{m}}$. By $[2,1.5]$ we know that $\operatorname{hd}_{R} S \geqq \operatorname{hd}_{R_{\mathfrak{m}}} S_{\mathfrak{m}}$ and therefore that $\operatorname{hd}_{R_{\mathfrak{m}}} S_{\mathfrak{m}}<\infty$. Applying $[2,3.8]$ we have

$$
\operatorname{hd}_{R_{\mathfrak{m}}} E_{\mathfrak{m}}=\operatorname{hd}_{R_{\mathfrak{m}}} S_{\mathfrak{m}}+\operatorname{hd}_{S_{\mathfrak{m}}} E_{\mathfrak{m}} .
$$

Therefore we have

$$
\operatorname{hd}_{R} E \geqq \operatorname{hd}_{R_{\mathfrak{m}}} E_{\mathfrak{m}}=\operatorname{hd}_{R_{\mathfrak{m}}} S_{\mathfrak{m}}+\mathrm{hd}_{S} E .
$$

Since $h_{R_{\mathrm{m}}} S_{\mathrm{m}} \geqq 0$ we have the first inequality. The second inequality follows immediately from 1.6. By $[2,3.9]$ we know that if rank $>0$, then $f$ contains at least one nonzero divisor. Consequently we know that $k_{\mathfrak{m}} \neq 0$ ([see 3 , VII, Exercise 9]). Since $R_{\mathfrak{m}}$ is a local ring and $S_{\mathfrak{m}}=R_{\mathfrak{m}} / k_{\mathfrak{m}}$, we have that hd ${ }_{R_{\mathfrak{m}}} S_{\mathfrak{m}}$ $>0$. The third and fourth inequalities now follow immediately from $\left(^{*}\right)$.

(2) We have recently shown that if $E$ is an $R$-module which has a finite resolution and if $\mathfrak{f}$, the annihilator of $E$, is not zero, then $f$ contains a nonzero divisor. Thus, in this proposition, if $S$ has a free finite $R$-resolution (e.g. if $R$ is a local ring), and $\mathfrak{f} \neq 0$, then it follows that rank $\mathfrak{f}>0$. (It should be observed that in [1, Appendix] one has to assume that has a finite free resolution.) 
Proposition 1.9. Let $S$ be the factor ring $R / \mathfrak{l}$ of $R$. Suppose that $h d_{R} S<\infty$ and that $E$ is an $S$-module such that $h d_{S} E<\infty$. Then we have

$$
h d_{R} E \leqq h d_{R} S+h d_{S} E \leqq 2 h d_{R} E .
$$

Proof. The first inequality is given us by [3, XVI, Exercise 5]. We know that there are maximal ideals $\mathfrak{m}$ and $\mathfrak{m}^{\prime}$ in $R$ such that $\mathrm{hd}_{S} E=\mathrm{hd}_{S_{(\mathfrak{m} / \mathfrak{l})}} E_{(\mathfrak{m} / \mathfrak{t})}$ and $\operatorname{hd}_{R} S=\operatorname{hd}_{R_{\mathfrak{m}^{\prime}}} S_{\mathfrak{m}^{\prime}}$. Since $\operatorname{hd}_{S_{\left(\mathfrak{m}^{\prime} / \mathfrak{t}\right)}} E_{\left(\mathfrak{m}^{\prime} / \mathfrak{l}\right)}<\infty$ and $\operatorname{hd}_{R_{\mathfrak{m}}} S_{\mathfrak{m}}<\infty$, we have by $[2,3.8]$ that

$$
\begin{aligned}
\operatorname{hd}_{R_{\mathfrak{m}}} E_{\mathfrak{m}} & =\operatorname{hd}_{R_{\mathfrak{m}}} S_{\mathfrak{m}}+\operatorname{hd}_{S_{(\mathfrak{m} / \mathfrak{t})}} E_{(\mathfrak{m} / \mathfrak{t})}, \\
\operatorname{hd}_{R_{\mathfrak{m}^{\prime}}} E_{\mathfrak{m}^{\prime}} & =\operatorname{hd}_{R_{\mathfrak{m}^{\prime}}} S_{\mathfrak{m}^{\prime}}+\operatorname{hd}_{S_{\left(\mathfrak{m}^{\prime} / \mathfrak{t}\right)}} E_{\left(\mathfrak{m}^{\prime} / \mathfrak{l}\right)} .
\end{aligned}
$$

But $\operatorname{hd}_{R} E \geqq \sup \left(h_{R_{\mathfrak{m}}} E_{\mathfrak{m}}, \operatorname{hd}_{R_{\mathfrak{m}^{\prime}}} E_{\mathfrak{m}^{\prime}}\right)$, from which it follows that 2 hd $_{R} E$ $\geqq \operatorname{hd}_{R} S+\operatorname{hd}_{S} E$.

We now give an example which shows that the right hand inequality in 1.9 can be an equality. Let $R=R^{\prime}+R^{\prime}$ (direct product) where $R^{\prime}$ is a regular local ring of dimension $n>0$, with maximal ideal m. Let $S=R^{\prime} / \mathrm{m}+R^{\prime}$ and $E=R^{\prime} / \mathfrak{m}+R^{\prime} / \mathfrak{m}$. Then $\mathrm{hd}_{R} E=\sup \left(\operatorname{hd}_{R^{\prime}} R^{\prime} / \mathfrak{m}, \mathrm{hd}_{R^{\prime}} R^{\prime} / \mathfrak{m}\right)=n$. On the other hand $\operatorname{hd}_{R} S=n$ and hd $E=n$. Thus $2 \operatorname{hd}_{R} E=\operatorname{hd}_{R} S+\operatorname{hd}_{S} E$.

Combining 1.6 and 1.9 we have

Proposition 1.10. Let $S$ be a factor ring of $R$ such that $h d_{R} S<\infty$. Then

$$
2 \operatorname{codim}_{R} R \geqq h d_{R} S+\operatorname{codim}_{S} S \text {. }
$$

Proposition 1.11. Let $E$ be an R-module. If $x_{1}, \cdots, x_{s}$ is an E-sequence, then

$$
\operatorname{codim}_{R} E \geqq s+\operatorname{codim}_{R /\left(x_{1}, \cdots, x_{s}\right)} E /\left(x_{1}, \cdots, x_{8}\right) E .
$$

If $x_{1}, \cdots, x_{s}$ is in the radical of $R$, then the above inequality becomes an equality.

Proof. Let $f: R \rightarrow R /\left(x_{1}, \cdots, x_{s}\right)$ be the natural homomorphism. The inequality follows immediately from the observation that if $f\left(y_{1}\right), \cdots, f\left(y_{t}\right)$ is an $E /\left(x_{1}, \cdots, x_{s}\right) E$-sequence, then $x_{1}, \cdots, x_{s}, y_{1}, \cdots, y_{t}$ is an $E$ sequence.

Let $y_{1}, \cdots, y_{t}$ be a maximal $E$-sequence in $R$. By 1.3 we know that there is a maximal ideal $\mathrm{m}$ in $R$ containing $\left(\mathfrak{f}, y_{1}, \cdots, y_{t}\right)$ where $\mathfrak{f}$ is the annihilator of $E$. Since the sequence $x_{1}, \cdots, x_{s}$ is contained in the radical of $R$, it is contained in $\mathrm{m}$. By 1.2 we know that all maximal $E$-sequences contained in $\mathrm{m}$ have the same length. Therefore there exist $z_{1}, \cdots, z_{t-s}$ in $m$ such that $x_{1}, \cdots, x_{s}, z_{1}, \cdots, z_{t-s}$ is a maximal $E$-sequence contained in $m$. Then $f\left(z_{1}\right), \cdots, f\left(z_{t-s}\right)$ is a maximal $E /\left(x_{1}, \cdots, x_{s}\right) E$-sequence contained in the maximal ideal $f(\mathfrak{m})$. Therefore we have the reverse inequality which gives us the desired equality. 
TheOREM 1.12. If $R\left[\left[X_{1}, \cdots, X_{n}\right]\right]$ is the ring of formal power series in the $n$-variables $X_{i}$, then

$$
\begin{aligned}
& \operatorname{codim}_{R\left[\left[X_{1}, \cdots, X_{n}\right]\right]} R\left[\left[X_{1}, \cdots, X_{n}\right]\right]=n+\operatorname{codim}_{R} R, \\
& \text { gl. } \operatorname{dim} R\left[\left[X_{1}, \cdots, X_{n}\right]\right]=n+\text { gl. } \operatorname{dim} R \text {. }
\end{aligned}
$$

Proof. It suffices to prove the theorem for the case $n=1$. It is well known that the ideal $(X)$ in $R[[X]]$ is in the radical of $R[[X]]$. It is also clear that $X$ is an $R[[X]]$-sequence. Since $R[[X]] /(X)=R$, the first equality follows immediately from 1.11 .

It follows from [3, II, Exer. 2] that $R[[X]]$ is a flat $R$-module, i.e. $\operatorname{Tor}_{p}^{R}(R[[X]], C)=0$ for all $p>0$ and all $R$-modules $C$. Also $R[[X]]$ contains $R$ as a direct summand. Thus we have that $\operatorname{hd}_{R} E=\operatorname{hd}_{R[[X]]} R[[X]] \otimes_{R} E$ for all $R$-modules $E$. Therefore if $\mathrm{gl} . \operatorname{dim} R=\infty$, then $\mathrm{gl} . \operatorname{dim} R[[X]]=\infty$.

Assume gl. $\operatorname{dim} R$ is finite. Let $f: R[[X]] \rightarrow R$ be the ring epimorphism $f\left(\sum_{i=0}^{\infty} a_{i} X^{i}\right)=a_{0}$. Since $X$ is in the Jacobson radical of $R[[X]]$, all the maximal ideals in $R[[X]]$ contain $X$. Therefore there is a one-one correspondence between the maximal ideals in $R[[X]]$ and those in $R$ given by $\mathfrak{m} \rightarrow f(\mathfrak{m})$. Given a maximal ideal $\mathfrak{m}$ in $R[[X]]$, the map $f$ induces a ring epimorphism $f_{\mathfrak{m}}: R[[X]]_{\mathfrak{m}} \rightarrow R_{f(\mathfrak{m})}$. Since $\operatorname{Ker} f_{\mathfrak{m}}=(X)$, we have $\mathrm{hd}_{R[[X]]_{\mathfrak{m}}} R_{f(\mathfrak{m})}$ $=1$. Also the fact that gl. $\operatorname{dim} R \geqq g l . \operatorname{dim} R_{f(\mathfrak{m})}[2,1.6]$, means that gl. $\operatorname{dim} R_{f(\mathfrak{m})}<\infty$. Combining this information with $[2,3.8]$ gives us that gl. $\operatorname{dim} R[[X]]_{\mathfrak{m}}=1+$ gl. $\operatorname{dim} R_{f(\mathfrak{m})}$. Since

gl. $\operatorname{dim} R[[X]]=\sup _{\mathfrak{m}} \mathrm{gl} \cdot \operatorname{dim} R[[X]]_{\mathfrak{m}}$ and $\operatorname{gl} \cdot \operatorname{dim} R=\sup _{f(\mathfrak{m})} \operatorname{gl} \cdot \operatorname{dim} R_{f(\mathfrak{m})}$

where $m$ runs through all maximal ideals in $R[[X]]$, we conclude that gl. $\operatorname{dim} R[[X]]=1+$ gl. $\operatorname{dim} R$.

2. Finitistic homological dimension. The modules considered in this section need not be finitely generated.

We define the weak homological dimension of the $R$-module $E$ as follows:

$$
-1 \leqq \mathrm{w} \cdot \operatorname{hd}_{R} E \leqq \infty
$$

where w. $\mathrm{hd}_{R} E<n$ if and only if $\operatorname{Tor}_{n}^{R}(E, C)=0$, for all $R$-modules $C$. If $E$ is finitely generated, then w. $\operatorname{hd}_{R} E=\mathrm{hd}_{R} E$ [3, VI, Exercise 3]. It follows easily from [3, V, Exercise 9] that if $\mathrm{w} . \mathrm{hd}_{R} E=n$, then $\operatorname{Tor}_{n}^{R}(E, R / \mathfrak{l}) \neq 0$ for some ideal $t$ in $R$.

LeMma 2.1. Let $E$ be an $R$-module and $S$ a multiplicatively closed subset of $R$ not containing 0 such that $E$ is uniquely divisible by every element in $S$. Then the natural homomorphism $E \rightarrow E_{S}$ is an isomorphism.

Proof. Since $E$ is uniquely divisible by every element in $S$, $\operatorname{Ker}\left(E \rightarrow E_{S}\right)$, which consists of all $e$ in $E$ such that $s e=0$ for some $s$ in $S$, is zero. Let $e / s$ be in $E_{S}$. Since $E$ is divisible by $s$ in $S$, there is an $e^{\prime}$ such that $s e^{\prime}=e$. There- 
fore $e^{\prime}$ considered as an element in $E_{S}$ is equal to $e / s$. Thus $E \rightarrow E_{S}$ is an epimorphism and consequently an isomorphism.

Proposition 2.2. Suppose $E$ is a nontrivial $R$-module with w. $h d_{R} E=n<\infty$. Let $\mathfrak{f}$ be a maximal ideal in $R$ such that $\operatorname{Tor}_{n}^{R}(E, R / \mathfrak{f}) \neq 0$. Then

(a) $\mathfrak{l}$ is a prime ideal in $R$.

(b) If $\mathfrak{l}$ is a maximal ideal in $R$, then $w$. $\operatorname{dim}_{R} E \leqq \operatorname{codim}_{R} R$.

(c) $\operatorname{Tor}_{n}^{R}(E, R / \mathfrak{l})$ is uniquely divisible by all elements not in $\mathfrak{t}$.

(d) $\operatorname{Tor}_{n}^{R}(E, R / \mathfrak{f})=\operatorname{Tor}_{n}^{R_{\mathfrak{t}}}\left(E_{\mathfrak{t}},(R / \mathfrak{f}) \mathfrak{t}\right)$.

(e) $w . \operatorname{dim}_{R} E=w . \operatorname{dim}_{R_{\mathfrak{l}}} E_{\mathfrak{l}} \leqq \operatorname{codim}_{R_{\mathfrak{l}}} R_{\mathfrak{t}}$.

Proof. (a) Suppose $\mathfrak{t}$ is not a prime ideal. Let $x$ be an element not in $\mathfrak{f}$ which is a zero-divisor for $R / \mathfrak{f}$. From the exact sequence

$$
0 \rightarrow(\mathfrak{f}, x) / \mathfrak{f} \rightarrow R / \mathfrak{l} \rightarrow R /(\mathfrak{f}, x) \rightarrow 0
$$

we deduce the exact sequence

$$
0 \rightarrow \operatorname{Tor}_{n}^{R}(E,(\mathfrak{l}, x) / \mathfrak{l}) \rightarrow \operatorname{Tor}_{n}^{R}(E, R / \mathfrak{l}) \rightarrow \operatorname{Tor}_{n}^{R}(E, R /(\mathfrak{f}, x)) .
$$

Since $(\mathfrak{f}, x)$ properly contains $\mathfrak{f}$, we have that $\operatorname{Tor}_{n}^{R}(E, R /(\mathfrak{f}, x))=0$. On the other hand $(\mathfrak{f}, x) / \mathfrak{f} \approx R / \mathfrak{l}^{\prime}$ where $\mathfrak{f}^{\prime}$ properly contains $\mathfrak{f}$, which means that $\operatorname{Tor}_{n}^{R}(E,(\mathfrak{f}, x) / \mathfrak{l})=0$. Therefore $\operatorname{Tor}_{n}^{R}(E, R / \mathfrak{t})=0$, which is a contradiction.

(b) Let $x_{1}, \cdots, x_{s}$ be a maximal $R$-sequence in $\mathfrak{f}$ and let $\mathfrak{f}^{\prime}=\left(x_{1}, \cdots, x_{s}\right)$. If $\mathfrak{f}=\mathfrak{f}^{\prime}$, then we have that $n \leqq \mathrm{hd}_{R} R / \mathfrak{t}^{\prime}=s \leqq \operatorname{codim}_{R} R$. Suppose $\mathfrak{f}^{\prime} \neq \mathfrak{f}$. Since by $1.2 \mathfrak{l}$ belongs to $\mathfrak{f}^{\prime}$, we know there is an ideal $\mathfrak{f}^{\prime \prime}$ in $R\left(\mathfrak{t}^{\prime \prime}=\mathfrak{l}^{\prime}: \mathfrak{t}\right)$ which properly contains $\mathfrak{l}^{\prime}$ and such that $\mathfrak{f} \cdot \mathfrak{f}^{\prime \prime}$ is contained in $\mathfrak{l}^{\prime}$. From the exact sequence

$$
0 \rightarrow \mathfrak{f}^{\prime \prime} / \mathfrak{l}^{\prime} \rightarrow R / \mathfrak{l}^{\prime} \rightarrow R / \mathfrak{l}^{\prime \prime} \rightarrow 0
$$

we deduce the exact sequence

$$
0 \rightarrow \operatorname{Tor}_{n}^{R}\left(E, \mathfrak{f}^{\prime \prime} / \mathfrak{l}^{\prime}\right) \rightarrow \operatorname{Tor}_{n}^{R}\left(E, R / \mathfrak{f}^{\prime}\right) .
$$

Since $\mathfrak{f}\left(\mathfrak{t}^{\prime \prime} / \mathfrak{f}^{\prime}\right)=0$, the module $\mathfrak{t}^{\prime \prime} / \mathfrak{t}^{\prime}$ is isomorphic to a finite direct sum of $R / \mathfrak{f}$. Thus we have that $\operatorname{Tor}_{n}^{R}\left(E, \mathfrak{f}^{\prime \prime} / \mathfrak{f}^{\prime}\right) \neq 0$ and consequently $\operatorname{Tor}_{n}^{R}\left(E, R / \mathfrak{l}^{\prime}\right)$ $\neq 0$. Therefore we again have $n \leqq \operatorname{codim}_{R} R$.

(c) Suppose $x$ in $R$ is not in $f$. From the exact sequence

$$
0 \rightarrow R / \mathfrak{f} \stackrel{f}{\rightarrow} R / \mathfrak{l} \rightarrow R /(\mathfrak{f}, x) \rightarrow 0
$$

where $f$ is multiplication by $x$, we deduce the exact sequence

$$
0 \rightarrow \operatorname{Tor}_{n}^{R}(E, R / \mathfrak{f}) \stackrel{g}{\rightarrow} \operatorname{Tor}_{n}^{R}(E, R / \mathfrak{f}) \rightarrow \operatorname{Tor}_{n}^{R}(E, R /(\mathfrak{f}, x))
$$

where $g$ is multiplication by $x$. Since $x$ is not in $\mathfrak{f}$, the ideal $(\mathfrak{l}, x)$ properly contains $\mathfrak{f}$ and $\operatorname{Tor}_{n}^{R}(E, R /(\mathfrak{l}, x))=0$. Thus $g$ is an isomorphism which means 
that $\operatorname{Tor}_{n}^{R}(E, R / \mathfrak{f})$ is uniquely divisible by $x$.

(d) It follows from (c) and 2.1 that $\operatorname{Tor}_{n}^{R}(E, R / \mathfrak{f})=\left(\operatorname{Tor}_{n}^{R}(E, R / \mathfrak{f})\right) \mathfrak{t}$. By [3, VII, Exercise 10] we have that $\left(\operatorname{Tor}_{n}^{R}(E, R / \mathfrak{t})\right) \mathfrak{t}=\operatorname{Tor}_{n}^{R_{\mathfrak{t}}}\left(E_{\mathfrak{l}},(R / \mathfrak{t}) \mathfrak{t}\right)$.

(e) By $[2,1.6]$ we know that w. $\operatorname{dim}_{R} E \geqq \mathrm{w} . \operatorname{dim}_{R \mathbb{t}} E_{\mathfrak{l}}$. The fact that w. $\operatorname{dim}_{R_{\mathfrak{t}}} E_{\mathfrak{t}} \geqq \mathrm{w} . \operatorname{dim}_{R} E$ follows immediately from (d). Since $(R / \mathfrak{t}) \mathfrak{t}=R_{\mathfrak{t}} / \mathfrak{f}_{\mathfrak{t}}$ and $\mathfrak{f}_{\mathfrak{t}}$ is the maximal ideal in $R_{\mathfrak{f}}$, it follows from (b) and (d) and what was just established that w. $\operatorname{dim}_{R_{\mathfrak{t}}} E_{\mathfrak{t}} \leqq \operatorname{codim}_{R_{\mathfrak{t}}} R_{\mathrm{t}}$.

We define the finitistic weak global dimension of $R$ as follows:

$$
0 \leqq \text { f.w. gl. } \operatorname{dim} R \leqq \infty
$$

where f. w. gl. $\operatorname{dim} R<n$ if and only if any $R$-module $E$ with w. $\operatorname{hd}_{R} E \geqq n$ has w. $\operatorname{hd}_{R} E=\infty$.

Proposition 2.3. Let $S$ be a multiplicatively closed subset of $R$ not containing zero. Then we have

$$
\text { f.w.gl. } \operatorname{dim} R \geqq f . w . g l . \operatorname{dim} R_{S} \geqq \operatorname{codim}_{R_{S}} R_{S} .
$$

Proof. Let $E$ be an $R_{S}$-module. By [3, VII, Exercise 10] we know w. $\operatorname{hd}_{R} E_{S}=$ w. $\operatorname{hd}_{R_{S}} E_{S}$. But by 2.1 we know that $E_{S}=E$. Therefore w. $\mathrm{hd}_{R} E$ $=\mathrm{w} . \mathrm{hd}_{R_{S}} E$, which gives the first inequality. The second inequality follows immediately from 1.6.

Theorem 2.4. For a ring $R$ we have f. w. gl. $\operatorname{dim} R=\sup _{\mathfrak{p}} \operatorname{codim}_{R_{\mathfrak{p}}} R_{\mathfrak{p}}$ $\leqq \operatorname{dim} R$ where $p$ runs through all prime ideals in $R$.

Proof. In order to establish the first relationship it suffices, in view of 2.3 , to prove that f. w. gl. $\operatorname{dim} R \leqq \sup _{\mathfrak{p}} \operatorname{codim}_{R_{\mathfrak{p}}} R_{\mathfrak{p}}$ where $\mathfrak{p}$ runs through all prime ideals in $R$. This is an immediate consequence of $2.2(\mathrm{e})$. The last inequality is well known.

Corollary 2.5. If $\operatorname{dim} R<\infty$, then there is a prime ideal $p$ in $R$ such that $\operatorname{codim}_{R_{\mathfrak{p}}} R_{\mathfrak{p}}=f . w . g l . \operatorname{dim} R_{\mathfrak{p}}=f . w . g l . \operatorname{dim} R$.

Since $\operatorname{dim} R<\infty$, there is a prime ideal $\mathfrak{p}$ in $R$ such that f. w. gl. $\operatorname{dim} R$ $=\operatorname{codim}_{R_{\mathfrak{p}}} R_{\mathfrak{p}}$. On the other hand we have by 2.3 that

f. w. gl. $\operatorname{dim} R \geqq$ f. w. gl. $\operatorname{dim} R_{\mathfrak{p}} \geqq \operatorname{codim}_{R_{\mathfrak{p}}} R_{\mathfrak{p}}$.

Therefore $\operatorname{codim}_{R_{\mathfrak{p}}} R_{\mathfrak{p}}=$ f. w. gl. $\operatorname{dim} R_{\mathfrak{p}}$.

We now give an example which shows that f. w. gl. $\operatorname{dim} R$ can be arbitrarily greater than $\operatorname{codim}_{R} R$. Let $R^{\prime}$ be a regular local ring such that $\operatorname{dim} R^{\prime}$ $=n \geqq 4$. Suppose the maximal ideal $\mathfrak{m}$ in $R^{\prime}$ is generated by $x_{1}, \cdots, x_{n}$. Let $R=R^{\prime} / \mathfrak{f}$ where $\mathfrak{f}=\left(x_{1}\right) \cap\left(x_{2}, \cdots, x_{n}\right)$. Since $R$ is a factor ring of a regular local ring, it satisfies the saturated chain condition on prime ideals $[2,2.8]$ and thus $\operatorname{dim} R=n-1$. Since $x_{1}+x_{2}$ is not a zero-divisor for $R / \mathfrak{t}$ and $\mathfrak{m}$ belongs to $\left(\mathfrak{l}, x_{1}+x_{2}\right)$, we have that $\operatorname{codim}_{R} R=1$. Let $\mathfrak{p}$ in $R$ be the prime ideal 
$\mathfrak{p}^{\prime} / \mathfrak{f}$ where $\mathfrak{p}^{\prime}=\left(x_{1}, \cdots, x_{n-1}\right)$. Then $R_{\mathfrak{p}}=\left(R^{\prime} /\left(x_{1}\right)\right)_{\mathfrak{p}^{\prime} /\left(x_{1}\right)}$ is a regular local ring of dimension $n-2$. Therefore $\operatorname{codim}_{R_{\mathfrak{p}}} R_{\mathfrak{p}}=n-2$, which gives us the desired example.

The following proposition is well known. However, for the sake of completeness we prove it.

Proposition 2.6. Let $R$ be a local ring such that dim $R \geqq 2$. Then $R$ has an infinite number of prime ideals of rank 1.

Proof. Suppose $R$ has only a finite number of prime ideals of rank 1 . Then there are only a finite number of prime ideals of rank $\leqq 1$. Let $p_{1}, \cdots, p_{n}$ be the prime ideals of rank $\leqq 1$. Since the maximal ideal $m$ of $R$ has rank $\geqq 2$, we know that $\mathfrak{m}$ is not contained in the union of the $\mathfrak{p}_{i}$. Let $x$ be in $\mathfrak{m}$ but not in any of the $\mathfrak{p}_{i}$. Then by Krull's principal ideal theorem rank $(x) \leqq 1$. Thus $x$ is contained in some $p_{i}$ which is a contradiction.

Lemma 2.7. Let $E$ be a nontrivial finitely generated $R$-module, $R$ an arbitrary ring. Let $x_{1}, \cdots, x_{n}$ be a sequence of elements in a prime ideal $p$. Then $x_{1}, \cdots, x_{n}$ in $R_{\mathfrak{p}}$ is an $E_{\mathfrak{p}}$-sequence if and only if $\mathfrak{p}$ does not contain the prime ideals $\mathfrak{p}_{i, 1}, \cdots, \mathfrak{p}_{i, h(i)}$ where the $\mathfrak{p}_{i, j}$ are the prime ideals belonging to (0) in $E /\left(x_{1}, \cdots, x_{i-1}\right) E$ which contain $x_{i}$.

Proof. The proof follows by standard arguments on primary decomposition of ideals and modules and their extensions to rings of quotients.

Proposition 2.8. If $1+\operatorname{codim}_{R} R \geqq \operatorname{dim} R, \operatorname{th} \in n f$. w. gl. $\operatorname{dim} R=\operatorname{codim}_{R} R$. If $R$ is a local ring, then this sufficient condition is also necessary.

Proof. If $\operatorname{codim}_{R} R=\infty$, then we have by $1.6(\mathrm{~b})$ and 2.4 that f. w. gl. $\operatorname{dim} R$ $=\infty$ and we are done. Suppose $\operatorname{codim}_{R} R=n<\infty$. Then $\operatorname{dim} R<\infty$ and there is a prime ideal $\mathfrak{p}$ in $R$ such that $\operatorname{codim}_{R_{\mathfrak{p}}} R_{\mathfrak{p}}=\mathrm{f}$. w. $\mathrm{gl}$. $\operatorname{dim} R$. Thus we have

$$
\operatorname{codim}_{R} R \leqq \operatorname{codim}_{R_{\mathfrak{p}}} R_{\mathfrak{p}} \leqq \operatorname{dim} R_{\mathfrak{p}} \leqq \operatorname{dim} R \leqq 1+\operatorname{codim}_{R} R .
$$

Thus if $\operatorname{codim}_{R} R<\operatorname{codim}_{R_{\mathfrak{p}}} R_{\mathfrak{p}}$, then $\operatorname{codim}_{R_{\mathfrak{p}}} R_{\mathfrak{p}}=\operatorname{dim} R_{\mathfrak{p}}=\operatorname{dim} R$ $=1+\operatorname{codim}_{R} R$. Since $\operatorname{dim} R_{\mathfrak{p}}=\operatorname{dim} R$, it follows that $\mathfrak{p}$ must be a maximal ideal in $R$. Applying 1.6 we have $\operatorname{codim}_{R} R \geqq \operatorname{codim}_{R_{\mathfrak{p}}} R_{\mathfrak{p}}$. But $\operatorname{codim}_{R_{\mathfrak{p}}} R_{\mathfrak{p}}$ $=1+\operatorname{codim}_{R} R$, which is a contradiction.

Suppose $R$ is a local ring and $\operatorname{codim}_{R} R \leqq \operatorname{dim} R-2$. Let $x_{1}, \cdots, x_{s}$ be a maximal $R$-sequence in $R$. Since $\operatorname{dim} R /\left(x_{1}, \cdots, x_{s}\right)=\operatorname{dim} R-\operatorname{codim}_{R} R$ $\geqq 2$, we have by 2.6 that there is a prime ideal $p$ containing $\left(x_{1}, \cdots, x_{8}\right)$ but which does not belong to $\left(x_{1}, \cdots, x_{s}\right)$. Then by 2.7 , we know that $x_{1}, \cdots, x_{s}$ in $R_{\mathfrak{p}}$ is an $R_{\mathfrak{p}}$-sequence. However this sequence is not maximal since $\mathfrak{p} R_{\mathfrak{p}}$ does not belong to the ideal $\left(x_{1}, \cdots, x_{\varepsilon}\right)$ in $R_{\mathfrak{p}}$. Thus we have f. w. gl. $\operatorname{dim} R \geqq \operatorname{codim}_{R_{\mathfrak{p}}} R_{\mathfrak{p}}>\operatorname{codim}_{R} R$. Therefore if f. w. gl. $\operatorname{dim} R$ $=\operatorname{codim}_{R} R$, then $1+\operatorname{codim}_{R} R \geqq \operatorname{dim} R$. 
Proposition 2.9. Let $R$ be a local ring and $p$ a prime ideal in $R$. If $x$ is a non-unit not in $\mathfrak{p}$, then there is a prime ideal $\mathfrak{p}^{*}$ such that $\mathfrak{p}^{*}$ contains $\mathfrak{p}$ but not $x$ and $\operatorname{dim} R / p^{*}=1$.

Proof. We proceed by induction on $\operatorname{dim} R / \mathfrak{p}$. It is clear that $\operatorname{dim} R / \mathfrak{p}>0$. If $\operatorname{dim} R / \mathfrak{p}=1$ we are done. Suppose $\operatorname{dim} R / \mathfrak{p}=k+1$ where $k \geqq 1$. Since $x$ is not a unit nor in $\mathfrak{p}, x+\mathfrak{p}$ is not a unit nor a zero-divisor in $R / p$. It follows therefore that $\operatorname{rank}(x+p)=1$. Since $\operatorname{dim} R / p \geqq 2$, we know by 2.6 that there are an infinite number of distinct ideals $p_{i}$ in $R$ which contain $p$ and such that rank $\left(p_{i} / \mathfrak{p}\right)=1$. Therefore if $x$ is in a $p_{i}$, the ideal $p_{i} / \mathfrak{p}$ belongs to $(x+\mathfrak{p})$. Thus only a finite number of $\mathfrak{p}_{i}$ contain $x$. Let $\mathfrak{p}^{\prime}$ be a $\mathfrak{p}_{i}$ which does not contain $x$. Since $\operatorname{dim} R / p^{\prime} \leqq k$, we know there is a $p^{*}$ containing $p$ but not $x$ and such that $\operatorname{dim} R / p^{*}=1$.

Theorem 2.10. Let $R$ be a local ring. Then f. w. gl. $\operatorname{dim} R=\sup _{\mathfrak{p}} \operatorname{codim}_{R_{\mathfrak{p}}} R_{\mathfrak{p}}$, where $\mathfrak{p}$ runs through all prime ideals such that $\operatorname{dim} R / \mathfrak{p} \leqq 1$.

Proof. Let $p$ be a prime ideal in $R$. Let $x_{1} / s_{1}, \cdots, x_{n} / s_{n}$ be an $R_{p}$-sequence in $R_{\mathfrak{p}}$. Then $x_{1}, \cdots, x_{n}$ in $R_{\mathfrak{p}}$ is an $R_{\mathfrak{p}}$-sequence. It follows from 2.7 that $\mathfrak{p}$ does not contain the prime ideals $\mathfrak{p}_{i, 1}, \cdots, \mathfrak{p}_{i, h(i)}$ where $\mathfrak{p}_{i, 1}, \cdots, \mathfrak{p}_{i, h(i)}$ are the prime ideals belonging to $\left(x_{1}, \cdots, x_{i-1}\right)$ which contain $x_{i}$. Let $x_{i, j}$ be in $\mathfrak{p}_{i, j}-\mathfrak{p}$ and let $x=\prod x_{i, j}$. Then by 2.9 there is a prime ideal $\mathfrak{p}^{*}$ which contains $\mathfrak{p}$ but not $x$ and such that $\operatorname{dim} R / \mathfrak{p}^{*}=1$. Applying 2.7 we have that since $\mathfrak{p}^{*}$ does not contain any of the $\mathfrak{p}_{i, j}$, the sequence $x_{1}, \cdots, x_{s}$ is an $R_{p^{*}}$-sequence in $R_{\mathfrak{p}^{*}}$. Thus we have $\operatorname{codim}_{R_{\mathfrak{p}^{*}}} R_{\mathfrak{p}^{*}} \geqq \operatorname{codim}_{R_{\mathfrak{p}}} R_{\mathfrak{p}}$. The desired equality follows immediately from this observation and 2.4 .

THEOREM 2.11. For an arbitrary ring $R$ we have f. w. gl. dim $R[X]$ $=\operatorname{codim}_{R(X)} R[X]=1 \mathrm{f} . w . g l . \operatorname{dim} R$.

Proof. Let $p$ be a prime ideal. Then by 2.7 there exists a sequence of elements $x_{1}, \cdots, x_{n}$ in $\mathfrak{p}$ such that $x_{1}, \cdots, x_{n}$ is a maximal $R_{\mathfrak{p}}$-sequence in $R_{\mathfrak{p}}$. As in the proof of 2.10 there is an element $s$ which is not in $p$ but in each of the prime ideals $\mathfrak{p}_{i, 1}, \cdots, \mathfrak{p}_{i, h(i)}$ where $\mathfrak{p}_{i, 1}, \cdots, \mathfrak{p}_{i, h(i)}$ are the prime ideals belonging to $\left(x_{1}, \cdots, x_{i-1}\right)$ which contain $x_{i}$. Then by $2.7, x_{1}, \cdots, x_{n}$ is an $R_{S}$-sequence in $R_{S}$ where $S=\left\{s^{n}\right\}$. Hence we have $\operatorname{codim}_{R_{S}} R_{S} \geqq \operatorname{codim}_{R_{\mathfrak{p}}} R_{\mathfrak{p}}$. Now $R_{S} \approx R[X] /(s X-1)$, and since $s X-1$ is not a zero-divisor in $R[X]$, we have $\operatorname{codim}_{R[X]} R[X] \geqq 1+\operatorname{codim}_{R_{S}} R_{S} \geqq 1+\operatorname{codim}_{R_{\mathfrak{p}}} R_{\mathfrak{p}}$. Since this is true for all prime ideals $\mathfrak{p}$ in $R$, it follows that $\operatorname{codim}_{R[X]} R[X] \geqq 1+\mathrm{f}$. w. gl. $\operatorname{dim} R$.

By $[5,6]$ we know that f. w. gl. $\operatorname{dim} R[X]=1+$ f. w. gl. $\operatorname{dim} R$. Since f. w. gl. $\operatorname{dim} R[X] \geqq \operatorname{codim}_{R[X]} R[X]$, we have

f. w. gl. $\operatorname{dim} R[X] \geqq \operatorname{codim}_{R[X]} R[X] \geqq 1+$ f. w. gl. $\operatorname{dim} R=$ f. w. $g l . \operatorname{dim} R[X]$.

Hence all the inequalities are equalities.

It should be noted that since given any $n \geqq 0$ there is a local ring $R$ such 
that f. w. gl. $\operatorname{dim} R-\operatorname{codim}_{R} R=n$ (see example after 2.5 ), we have by 2.11 that given any $n \geqq 1$, there exist rings $R$ such that $\operatorname{codim}_{R[X]} R[X]-\operatorname{codim}_{R} R$ $=n$. However while f. w. gl. $\operatorname{dim} R>\operatorname{codim}_{R} R$, we have f. w. gl. $\operatorname{dim} R[X]$ $=\operatorname{codim}_{R[X]} R[X]$.

3. Unique factorization in local rings. We first prove a rather elementary lemma.

Lemma 3.1. Let $R$ be an integral domain. Then the following conditions are both necessary and sufficient for $R$ to be a unique factorization domain:

(a) $R$ satisfies the ascending chain condition for principal ideals.

(b) The intersection of any two principal ideals in $R$ is again a principal ideal.

Proof. That these conditions are necessary is easy to see. Hence we will prove sufficiency. If we show that every pair of elements has a greatest common divisor, the result will follow from [6, p. 119].

Let $x, y \in R$. Then $(x) \cap(y)=(c)$, and $c$ is the least common multiple of $x$ and $y$. Now let $x y=d c$. We show that $d$ is the g. c. d. of $x$ and $y$.

We have $c=r x=s y$. Hence $x y=d c=d r x=d s y$. Thus $y=d r, x=d s$ so $d$ is a common divisor. Let $d^{\prime}$ be a common divisor of $x$ and $y$. Then $x=r^{\prime} d^{\prime}$, $y=s^{\prime} d^{\prime}$. Since $r^{\prime} s^{\prime} d^{\prime}$ is a common multiple of $x$ and $y$, we have that $c$ divides $r^{\prime} s^{\prime} d^{\prime}$. Also, since $x y=\left(r^{\prime} s^{\prime} d^{\prime}\right) d^{\prime}=d c$, we have $d^{\prime}$ divides $d$. Thus $d$ is the g. c. d. of $x$ and $y$.

Proposition 3.2. Let $R$ be a noetherian integral domain with the property that every finitely generated projective $R$-module is free. Then a necessary and sufficient condition that $R$ be a unique factorization domain is that $h d_{R} R /(x, y)$ $\leqq 2$ for every pair of elements $x, y \in R$.

Proof. Consider the exact sequence

$$
0 \rightarrow K \rightarrow F \stackrel{g}{\rightarrow}(x, y) \rightarrow 0
$$

where $F$ is free on two generators $z_{1}, z_{2}$ and $g$ is defined by $g\left(z_{1}\right)=x, g\left(z_{2}\right)=y$. Then $K=\left\{r_{1} z_{1}+r_{2} z_{2} \mid r_{1} x+r_{2} y=0\right\}$. It is clear that $K \approx(x) \cap(y)$.

Now, if $\operatorname{hd}_{R} R /(x, y) \leqq 2$, then $\operatorname{hd}_{R}(x, y) \leqq 1$, so that $K$ is projective, hence free due to our assumption about $R$. Thus $(x) \cap(y)$ is a free ideal in $R$ and must therefore be principal. Thus the condition is sufficient.

Conversely, if $R$ is a unique factorization domain, then $(x) \cap(y)$ is principal for all $x, y \in R$. Therefore $K$ is free and $\operatorname{hd}_{R} R /(x, y) \leqq 2$.

Corollary 3.3. A necessary and sufficient condition that a local integral domain $R$ be a unique factorization domain is that $h d_{R} R /(x, y) \leqq 2$ for every pair of elements $x, y \in R\left({ }^{3}\right)$.

(3) It is unnecessary to assume that the local ring $R$ is an integral domain. 
Proposition 3.4. Let $R$ be a local integral domain, and $\hat{R}$ its completion. If $\hat{R}$ is a unique factorization domain, then so is $R$.

Proof. Let $x, y \in R$. We must show that $\operatorname{hd}_{R} R /(x, y) \leqq 2$. By $[2,3.2]$, we know that $\operatorname{hd}_{R} R /(x, y)=\operatorname{hd}_{\widehat{R}} \hat{R} /(x, y)$. Thus, if $\hat{R}$ is a unique factorization domain, we have hd $\widehat{R} \hat{R} /(x, y) \leqq 2$ and we are done.

ThEOREM 3.5. Every nonramified regular local ring is a unique factorization domain.

Proof. Let $R$ be a nonramified regular local ring. Then $\hat{R}$ is complete, nonramified and regular. By $[4,1.8]$, we know that $\hat{R}$ is a unique factorization domain and so by 3.4 we see that $R$ is also. (This theorem is due to Krull [7].)

We observe that this gives a new proof that every regular geometric local ring is a unique factorization domain.

\section{REFERENCES}

1. M. Auslander, On the dimension of modules and algebras III, Nagoya Math. J. vol. 9 (1955) pp. 67-77.

2. M. Auslander and D. Buchsbaum, Homological dimension in local rings, Trans. Amer. Math. Soc. vol. 85 (1957) pp. 390-405.

3. H. Cartan and S. Eilenberg, Homological algebra, Princeton, 1955.

4. I. S. Cohen, On the structure and ideal theory of complete local rings, Trans. Amer. Math. Soc. vol. 59 (1946) pp. 54-106.

5. S. Eilenberg, A. Rosenberg and D. Zelinsky, On the dimension of modules and algebras, VIII, Nagoya Math. J. vol. 12 (1957) pp. 71-93.

6. N. Jacobson, Lectures in abstract algebra, D. van Nostrand, 1951.

7. W. Krull, Zur Theorie der kommutativen Integritätsbereiche, J. Reine Angew. Math. vol. 192 (1953) pp. 230-252.

Institute for Advanced Study,

PRINCETON, N. J.

Brown University,

Providence, R. I. 